\title{
Correction to: Changes in maximum lip-closing force after extraction and nonextraction orthodontic treatments
}

\author{
Tae-Hyun Choi ${ }^{\mathrm{a}}$, So-Hyun Kim ${ }^{\mathrm{a}}$, Cheul Kim ${ }^{\mathrm{b}}$, Yoon-Ah Kook ${ }^{\mathrm{c}}$, Brent E. Larson ${ }^{\mathrm{d}}$, Nam-Ki Lee \\ ${ }^{a}$ Department of Orthodontics, Seoul National University Bundang Hospital, Section of Dentistry, Seoul National University, Seongnam, Korea \\ ${ }^{b}$ Department of Oral Medicine and Diagnosis, College of Dentistry and Research Institute of Oral Science, Gangneung-Wonju National University, \\ Gangneung, Korea \\ 'Department of Orthodontics, Seoul St. Mary's Hospital, The Catholic University of Korea, Seoul, Korea \\ ${ }^{d}$ Division of Orthodontics, School of Dentistry, University of Minnesota, Minneapolis, MN, USA
}

https://doi.org/10.4041/kjod.2020.50.2.120

Korean J Orthod 2020;50(2):120-128

To the Editor;

In the above article, we found that our affiliation was published incorrectly.

Tae-Hyun Choi ${ }^{\mathrm{a}}$, So-Hyun Kima ${ }^{\mathrm{a}}$, and Nam-Ki Lee

${ }^{a}$ Department of Orthodontics, Seoul National University Bundang Hospital, Section of Dentistry, Seoul National University, Seongnam, Korea

Corresponding author: Nam-Ki Lee.

Professor, Department of Orthodontics, Seoul National University Bundang Hospital, Section of Dentistry, Seoul National University, 82 Gumi-ro 173beon-gil, Bundang-gu, Seongnam 13620, Korea.

Tel +82-31-787-2787 e-mail: ortho0328@naver.com

This should be corrected as follows;

Tae-Hyun Choi ${ }^{\mathrm{a}}$, So-Hyun Kimª and Nam-Ki Lee ${ }^{\mathrm{a}}$

${ }^{a}$ Department of Orthodontics, Section of Dentistry, Seoul National University Bundang Hospital, Seongnam, Korea

Corresponding author: Nam-Ki Lee.

Professor, Department of Orthodontics, Section of Dentistry, Seoul National University Bundang Hospital, 82 Gumi-ro 173beongil, Bundang-gu, Seongnam 13620, Korea.

Tel +82-31-787-2787 e-mail: ortho0328@naver.com

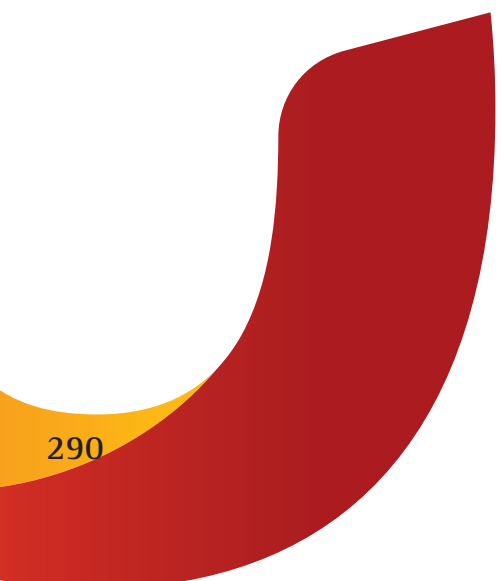

(C) 2020 The Korean Association of Orthodontists.

This is an Open Access article distributed under the terms of the Creative Commons Attribution Non-Commercial License (http://creativecommons.org/licenses/by-nc/4.0) which permits unrestricted non-commercial use, distribution, and reproduction in any medium, provided the original work is properly cited. 